\title{
Evaluation of Body Surface Area Formulae Based on 3D Body Scans
}

\author{
Grzegorz REDLARSKI, Marek KRAWCZUK, Aleksander PALKOWSKI* \\ Gdansk University of Technology, Department of Mechatronics and High Voltage Engineering, \\ Gdansk, Poland
}

DOI: $10.15221 / 17.054$

http://dx.doi.org/10.15221/17.054

\begin{abstract}
Human body surface area (BSA) is an established parameter for the calculation of chemotherapy drugs dosage, treatment of chronic hepatitis $\mathrm{B}$, treatment of burns or for establishing a dosing regimen for antimicrobials. Although being a critical parameter, usage of modern 3D scanners, which would measure the exact BSA value, is often impossible in time-sensitive operations or for patients unable to withstand the scanning process. Therefore, over the last decades considerable research efforts have been devoted to development of simple formulae for BSA approximation. The formulae use a small number of state variables (weight, height, age, sex), which are intended to be easily obtained for every patient. The formulae parameters were estimated independently for isolated groups of subjects, which should rise suspicion whether any of the formulae used is indeed effective and safe for medical treatment. Here, we provide an extended analysis of 43 BSA formulae based on 152 patients scanned with a hand-held 3D scanner. Upon comparison of the real BSA values with estimations made by the formulae, we can conclude that most of the formulae exhibit a high relative BSA error, ranging from $9.83 \%$ to $43.27 \%$.
\end{abstract}

Keywords: anthropometry, body surface area, dosing regimen, medical sciences, oncology, whole body 3D model

\section{Introduction}

Human body surface area (BSA) is a parameter commonly used in medicine, mainly in oncology and burns treatment [1], [2]. It is crucial to determine the exact BSA value of the patient with minimal error using only the knowledge of patient's height and weight. Height and weight can be relatively easy to obtain especially when working under time pressure, which makes those factors suitable for medical examination. The existing methods used to compute BSA prove to be inaccurate and may cause ineffective chemotherapy or burns treatment.

Due to technical difficulties pertinent to precise measurement of the BSA value (especially in time-sensitive medical procedures) considerable research efforts have been devoted to development of simple formulae for BSA approximation. Starting with Meeh's research on the matter [3], the past century led to the development of over 40 formulae tuned up to specific test groups.

In most medical cases, the DuBois formula [4] is most commonly used. However, the medical community to this day has no agreement which of the formulae known is the most precise and safe.

The latest sources [5], [6], [7] documenting the validity of the calculation of drug doses for chemotherapy based on the BSA have emphasized its most important benefit, which is an increased patient survival rate. The need to rely on the BSA in the near future is also obvious. Nevertheless, there is also a well-known series of studies that have reported on severe consequences resulting from the incorrect use of the BSA as a measure for drug dosing [7], [8], [9], [10]. Strong scientific evidence of its limitations has been provided, followed by a statement that the need to develop appropriate procedures will be one of the most significant challenges in the nearest future.

Here, we provide an extended analysis of the majority of known BSA formulae and their approximation errors based on 152 volunteers examined. The results reveal that the today's approach to the development of BSA formulae has significant drawbacks and should be revised.

Results shown in the paper indicate that there is a necessity to create supporting methods for selecting the best BSA calculation method. The need is particular in case of people with abnormal physique, i.e., people underweight, overweight or people with other major, noticeable deformities and diseases causing anatomical abnormalities.

\footnotetext{
*aleksander.palkowski@pg.gda.pl; +48-58-347-1894
} 


\section{Methods}

\subsection{Study design}

The main objective of the study is to investigate errors in BSA calculation for 43 known formulae. In order to accomplish this, the body surface of 152 volunteers forming an heterogeneous group has been scanned using a modern 3D scanner. Each raw scan required also a series of post-processing methods to be used for the data to be exported properly.

The study was approved by Independent Bioethics Commission for Research at Medical University of Gdansk. Written informed participation consent was obtained from all the volunteers taking part in the procedure. All experiments presented were carried out in accordance with the approved guidelines.

\subsection{Study population}

The study presents results obtained from scanning 152 adult volunteers of various sex, age, and physique. The group includes young and healthy subjects from college communities, as well as older people suffering from diseases that strongly influence the body structure, e.g., anorexia or obesity.

The volunteers are divided into two groups based on their body mass index (BMI), indicating their body structure. The first group consists of subjects with BMI in range $18.5 \mathrm{~kg} / \mathrm{m}^{2}-25 \mathrm{~kg} / \mathrm{m}^{2}$, the second consists of subjects with $\mathrm{BMI}$ in other ranges. Table 1 shows the most relevant characteristics of the study group.

Table 1. Characteristics of the volunteers.

\begin{tabular}{|l|l|l|l|}
\hline Characteristics & $\begin{array}{l}\text { All volunteers } \\
(\mathrm{n}=152)\end{array}$ & $\begin{array}{l}18.5 \leq \mathrm{BMI}<25 \\
(\mathrm{n}=79)\end{array}$ & $\begin{array}{l}18.5>\mathrm{BMI} \geq 25 \\
(\mathrm{n}=73)\end{array}$ \\
\hline Age-yrs. & $29.03 \pm 11.77$ & $24.06 \pm 6.38$ & $34.41 \pm 13.79$ \\
\hline Age below 40-no. (\%) & $125(82.2 \%)$ & $77(97.5 \%)$ & $48(65.8 \%)$ \\
\hline Male sex-no. (\%) & $111(73.0 \%)$ & $65(82.3 \%)$ & $46(63.0 \%)$ \\
\hline BSA-m ${ }^{2}$ & $1.986 \pm 0.231$ & $1.871 \pm 0.151$ & $2.111 \pm 0.238$ \\
\hline Weight-kg & $83.00 \pm 21.86$ & $69.56 \pm 8.93$ & $97.54 \pm 22.44$ \\
\hline Height-cm & $175.77 \pm 8.60$ & $177.15 \pm 7.59$ & $174.27 \pm 9.40$ \\
\hline
\end{tabular}

\subsection{Scanning process}

The volunteers were obligated to take part in a whole-body scanning process. The Artec 3D Eva handheld scanner have been used as the measurement device (Fig. 1a). The scanning process was conducted in an upright position and, in cases of severe physical disfunctionalities or limb scanning, was conducted seated.

A total of five scans were performed for every subject-four for each limb and one for the main body part. The resulting body model (Fig. 1b) was obtained through a series of graphical processing methods. The entirety of the process was conducted with the Artec Studio 11 Professional software. First, the points cloud obtained from the scan was subjected to a global registration process, which normalized the points locations. Secondly, the normalized point cloud was subjected to a fusion process resulting in a solid and wireframe representation of the model. And lastly, a series of post-processing methods, such as small-object filter, hole filling, and smoothing, were used on the solid model to improve its quality. The BSA measure was calculated by the software based on surface of the 3D model.

\subsection{Body surface area formulae}

Since 1879 many BSA calculation formulae were developed. To determine the BSA value these formulae use the patient's weight (in kilograms) and in most cases also the patient's height (in centimeters).

The early BSA calculation formulae were developed using different coating methods in order to obtain the patient's real BSA value. The accuracy of the methods depended not only on the type of mathematical approximation, but also on the quality of the measuring methods. In the process of obtaining real BSA values, the authors used subjects from a limited range of race, sex, and age, often generalizing the outcome BSA formula for an entire population.

In modern times, different methods of obtaining real BSA values were used. Still, when using the BSA calculation formulae the computed BSA values for the same patient vary in an extensive way. This should not surprise when using old calculation formulae, but this regularity is true even for modern formulae (Fig. 2). 

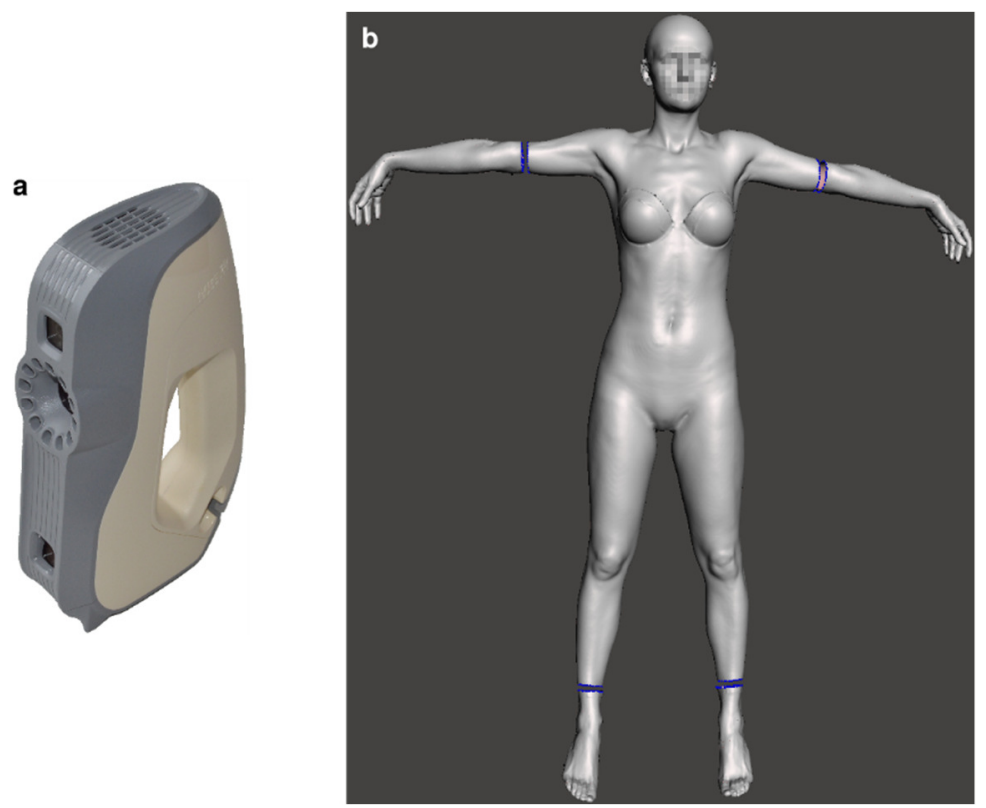

Fig. 1. (a) Artec 3D Eva scanner used as the scanning device. (b) An example of a 3D model of a patient's body.
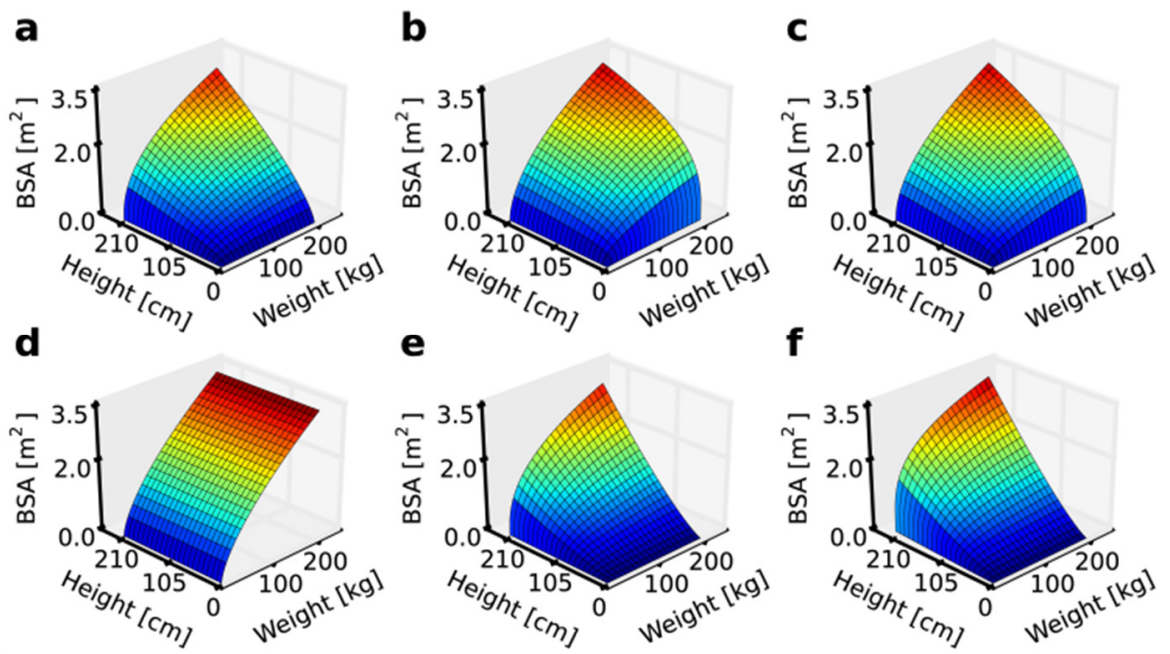

Fig. 2. Examples of BSA surfaces in accordance with various formulae. (a) DuBois and DuBois.

(b) Boyd \#1. (c) Gehan and George. (d) Livingston and Lee. (e) Schlich. (f) Nwoye [2].

Currently, in an over a century-old history of the study of reliable determination of BSA, we can find over 40 mathematical formulae based on a number of state variables. Table 2 presents the most popular formulae used-the Boyd, Du Bois and Du Bois, Gehan and George, Haycock et al., and Mosteller formulae [11]. However, for the purpose of further analysis, we will consider all 43 formulae found in the literature.

Table 2. The most popular BSA formulae used by the medical community.

\begin{tabular}{|l|l|l|}
\hline Authors (year) & Formula & Reference \\
\hline Du Bois and Du Bois (1916) & $0.007184 \times W^{0.425} \times H^{0.725}$ & 4 \\
\hline Boyd \#1 (1935) & $\begin{array}{l}0.0003207 \times(W \times 1000)^{0.7285-0.0188 \times \log _{10}(W \times 1000)} \\
\times H^{0.3}\end{array}$ & 12 \\
\hline Gehan and George (1970) & $0.0235 \times W^{0.51456} \times H^{0.42246}$ & 13 \\
\hline Haycock et al. (1978) & $0.024265 \times W^{0.5378} \times H^{0.3964}$ & 14 \\
\hline Mosteller (1987) & $\sqrt{W \times H / 3600}$ & 15 \\
\hline
\end{tabular}




\section{Results}

The formulae are characterized by a high correlation of BSA with limited state variables for a representative sample of people, determined by the subjects enrolled therein. However, if confronted with an unknown hitherto and heterogeneous group of subjects, the formulae accuracy drops (Fig. 3). While being mostly accurate for patients of BMI in the normal range (Fig. 3a), the BSA formulae under consideration give far higher error for other ranges of BMI, i.e., for underweighted or overweighed people (Fig. 3b).

The results shown in Fig. 3a indicate that most known formulae are well suited for calculating BSA values for people with normal physique $\left(18.5 \mathrm{~kg} / \mathrm{m}^{2} \leq \mathrm{BMI}<25 \mathrm{~kg} / \mathrm{m}^{2}\right)$. However, this is not the case for other ranges of $\mathrm{BMI}$ where the median error is still above $1 \%$ and the maximum-to-minimum error span is equal to $6.49 \%$ for the best case.

To give a detailed example, Fig. 4 presents BSA calculation errors for one chosen subject. The subject is a 63-old male with weight $124 \mathrm{~kg}$ and height $163 \mathrm{~cm}\left(\mathrm{BSA}=2.395 \mathrm{~m}^{2}\right)$, who can be considered as very severely obese $\left(B M l=46.7 \mathrm{~kg} / \mathrm{m}^{2}\right)$. One can see how inconsistent the errors are, i.e., they span from about $-8 \%$ (the estimation is lower than the real value) to above $27 \%$. The best formula for this case - the Wang and Hihara formula — cannot be considered as the best formula overall by judging from the analysis presented in Fig. 3. This is true for several other formulae, e.g., for the Lowe, Milazzo or Anderson et al. formulae.

However, in almost all cases we can choose formulae which are always inaccurate, e.g., the Meeh, Vierordt, Bardeen, Bierring or Boyd \#2 formulae.

a

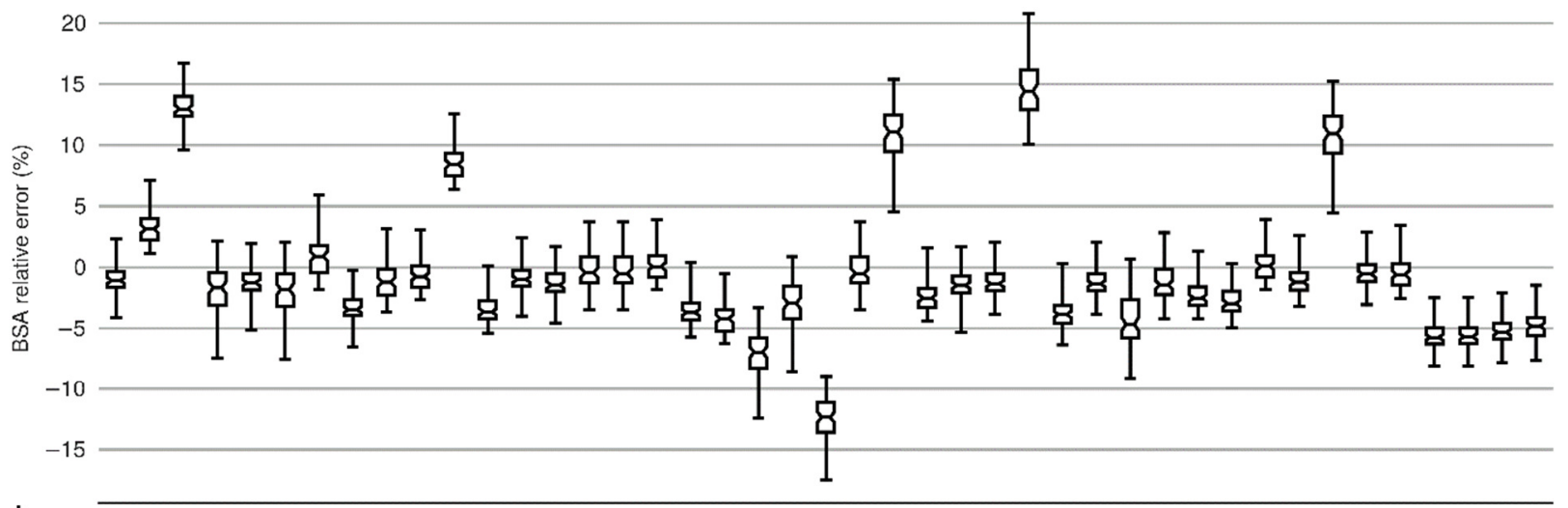

b
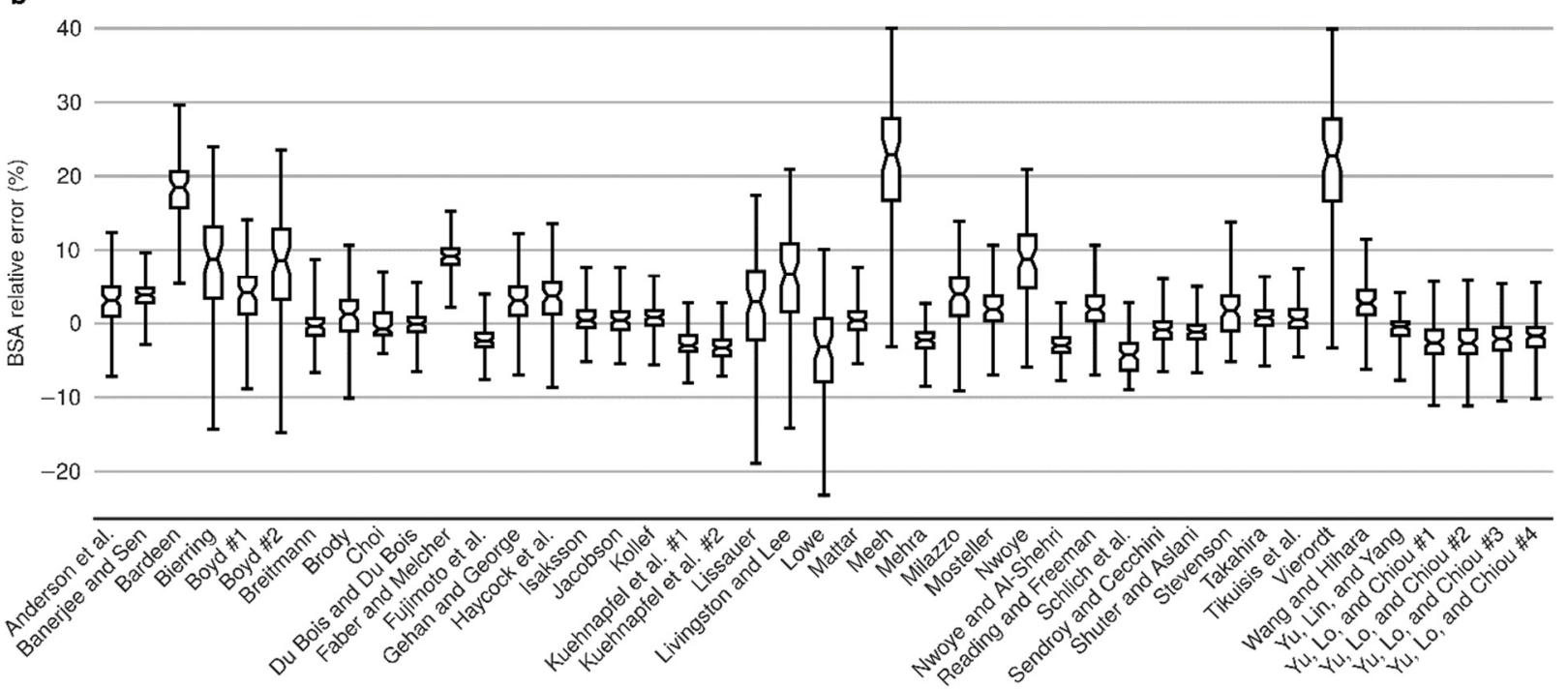

Fig. 3. Relative error of BSA estimation by the known formulae. Shown are relative percentage errors between BSA values measured and those calculated by the formulae known so far. (a) Error distribution for patients characterized by BMI greater or equal to $18.5 \mathrm{~kg} / \mathrm{m}^{2}$ and lesser than $25 \mathrm{~kg} / \mathrm{m}^{2}$. (b) Error distribution for patients characterized by $B M I$ of other ranges. 


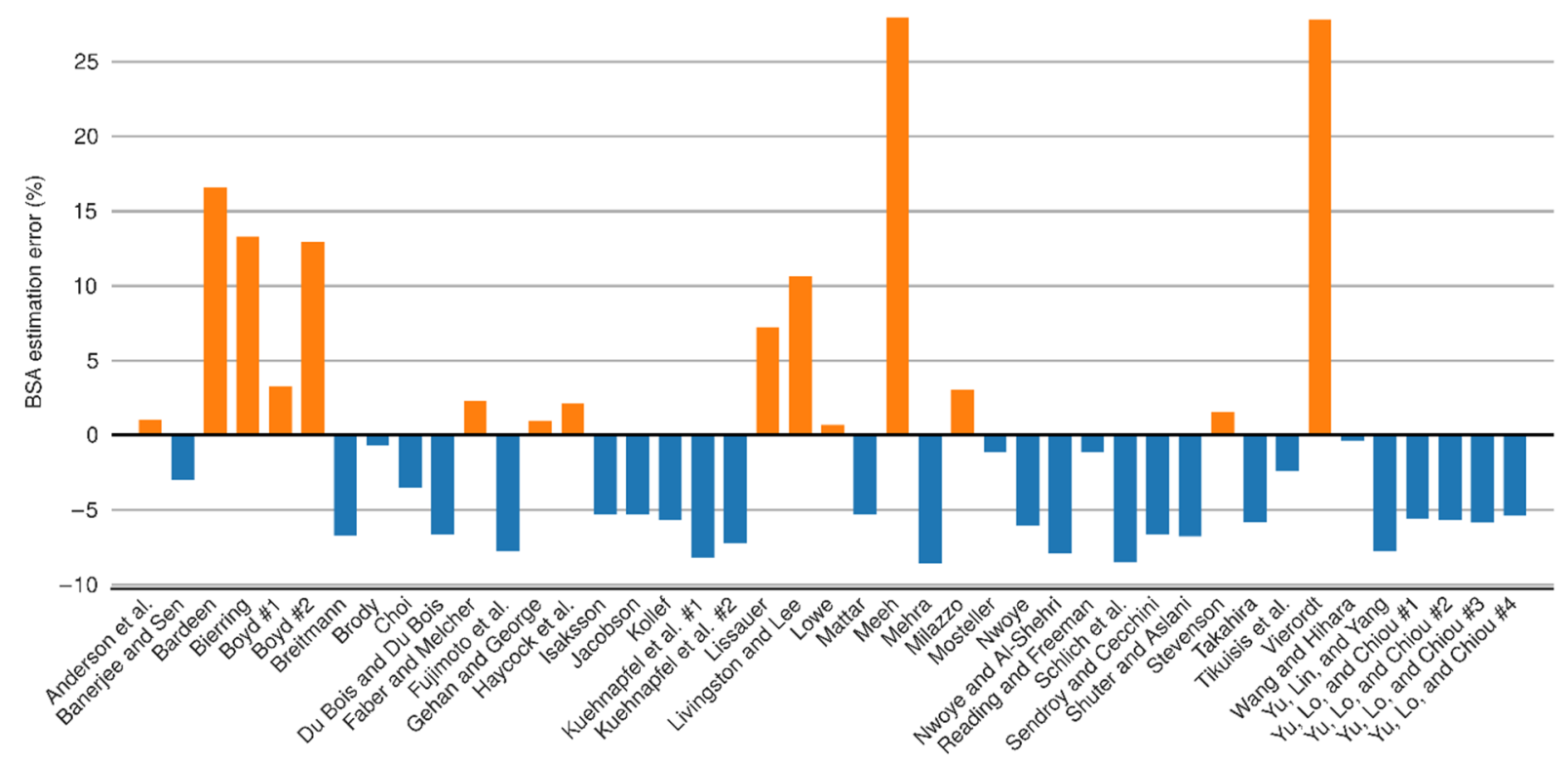

Fig. 4. Percentage error between the real BSA and BSA values calculated using the formulae under consideration for a patient with weight $124 \mathrm{~kg}$ and height $163 \mathrm{~cm}\left(B S A=2.395 \mathrm{~m}^{2}\right)$.

\section{Conclusions}

The paper presents and extended analysis of 43 BSA formulae based on real BSA values obtained from 152 volunteers scanned by a modern hand-held 3D scanner. The results show that almost all formulae under consideration are heavily inconsistent and produce high error ranges even for a limited group of subjects. This is especially noticeable for people with abnormal physique (18.5 $>\mathrm{BMI} \geq 25$ ). There are, however, formulae that give fairly good estimation for most cases, e.g., the Du Bois and Du Bois formula.

Maybe the most important conclusion from the analysis presented in the paper is how BSA estimation based on the formulae presented may impact medical procedures and, ultimately, patients' chance of survival. Certain medical procedures are in fact life-saving, thus a clear connection between BSA miscalculation and patients' death due to misuse of treatment can be drawn. It should be clearly stated that the exact BSA calculation is one of the most important issues in the processes that support the effectiveness of the treatment of numerous diseases, primarily those that, if untreated or treated incorrectly, lead to the death of patients. The currently used formulae for the determination of the BSA, including the latest ones, not only do not allow for the precise determination of the body surface area but also provide no information concerning which one, in relation to the individual variability of patients, leads to the most accurate results.

\section{Acknowledgments}

This work was supported by funds from the Polish National Science Centre (2014/15/B/NZ7/01018).

\section{References}

[1] G. Redlarski and P.M. Tojza, "Computer Supported Analysis of the Human Body Surface Area", International Journal of Innovative Computing, Information and Control, Vol.9, No.5, 2013, pp. 1801-1818.

[2] G. Redlarski, A. Palkowski and M. Krawczuk, "Body surface area formulae: an alarming ambiguity", Sci Rep, Vol.6, 2016, p. 27966, http://dx.doi.org/10.1038/srep27966.

[3] K. Meeh, "Oberflächenmessungen des menschlichen Körpers", Z Biol, Vol.15, 1879, pp. 425-458.

[4] D. Du Bois and E.F. Du Bois, "A formula to estimate the approximate surface area if height and weight be known", in Arch Intern Med, Vol.17, 1916, pp. 863-871.

[5] T. Kouno, N. Katsumata, H. Mukai, M. Ando and T. Watanabe, "Standardization of the Body Surface Area (BSA) Formula to Calculate the Dose of Anticancer Agents in Japan", Jpn. J. Clin. Oncol, Vol.33, 2003, pp. 309-313. 
[6] A. Gerina-Berzina, U. Vikmanis, U. Teibe and S. Umbrashko, "Anthropometric measurements of the body composition of cancer patients determine the precise role of the body surface area and the calculation of the dose of chemotherapy", Pap. Anthropol, Vol.21, 2012.

[7] S.D. Baker et al., "Role of Body Surface Area in Dosing of Investigational Anticancer Agents in Adults, 1991-2001", J. Natl. Cancer Inst, Vol.94, 2002, pp. 1883-1888, http://dx.doi.org/10.1093/jnci/94.24.1883.

[8] J.H. Beumer, E. Chu and S.J. Salamone, "Body-Surface Area-Based Chemotherapy Dosing: Appropriate in the 21st Century?", J. Clin. Oncol, Vol.30, 2012, pp. 3896-3897, http://dx.doi.org/10.1200/JCO.2012.44.2863.

[9] J.J. Griggs et al., "Appropriate Chemotherapy Dosing for Obese Adult Patients With Cancer: American Society of Clinical Oncology Clinical Practice Guideline", J. Clin. Oncol, Vol.30, 2012, pp.1553-1561, http://dx.doi.org/10.1200/JCO.2011.39.9436.

[10]A.A. Miller et al., "Prospective Evaluation of Body Surface Area as a Determinant of Paclitaxel Pharmacokinetics and Pharmacodynamics in Women with Solid Tumors: Cancer and Leukemia Group B Study 9763", Clin. Cancer Res, Vol.10, 2004, pp. 8325-8331, http://dx.doi.org/10.1158/1078-0432.CCR-04-1078.

[11]W. Faisal, H.-M. Tang, S. Tiley and C. Kukard, "Not All Body Surface Area Formulas Are the Same, but Does It Matter?", J Glob Oncol, Vol.2, No.6, 2016, pp. 436-437, http://dx.doi.org/10.1200/JGO.2016.005876.

[12] E. Boyd, The Growth of the Surface Area of the Human Body, Minneapolis MN, USA, University of Minnesota Press, 1935.

[13]E.A. Gehan and S.L. George, "Estimation of human body surface area from height and weight", in Cancer Chemother Reports, Vol.54, 1970, pp. 225-235.

[14] G.B. Haycock, G.J. Schwartz and D.H. Wisotsky, "Geometric method for measuring body surface area: A height-weight formula validated in infants, children, and adults", in J Pediatr, Vol.93, 1978, pp. 62-66, http://dx.doi.org/10.1016/S0022-3476(78)80601-5.

[15] R.D. Mosteller, "Simplified Calculation of Body-Surface Area", in N Engl J Med, Vol.317, 1987, p. 1098, http://dx.doi.org/10.1056/NEJM198710223171717. 